Effective in 2014, the CPD programme for SAJSM will be administered by Medical Practice Consulting:

CPD questionnaires must be completed online at www.mpconsulting.co.za

A maximum of 3 CEUs will be awarded per correctly completed test.

\title{
TRUE OR FALSE
}

Common acute and chronic musculoskeletal injuries among female adolescent field hockey players in KwaZulu-Natal, South Africa (SA)

1. The majority of field hockey musculoskeletal injuries are to the upper extremities.

2. In field hockey, goalkeepers have the highest rate of injury.

3. Chronic lower-back injuries in field hockey players have been associated with hip flexor contractures.

Collagen gene interactions and endurance running performance

4. There are $\sim 20$ known gene variants associated specifically with endurance performance.

5. Both type V and VI collagen regulate collagen fibrillogenesis.

6. Increased type $\mathrm{V}$ collagen production alters the mechanical properties of the tissue, leading to decreased endurance performance.

7. The COL $3 A 1$ and COL12A1 genes serve as potential markers for endurance running performance.

Steps that count! A feasibility study of a pedometer-based health promotion intervention in an employed, SA population

8. Pedometers have become popular for use in physical activity interventions in various settings to facilitate behavioural change.

9. Goal-setting, feedback on progress and strategies for overcoming barriers are common features for successful interventions to increase the volume of walking.

Micro-oscillations in positive and negative affect during competitive laboratory cycle time trials

10. Athletes were more likely to maintain or increase exercise intensity when positive affect was experienced.
11. Levels of positivity and negativity reported by participants during exercise were shown to oscillate, providing evidence for the dynamic, emotive nature of sport performance.

12. High levels of negative affect and lower levels of positive affect were associated with better performances by the cyclists.

13. In self-regulation theory, monitoring of self- and norm-referenced information enables individuals to make the necessary adjustments to minimise the discrepancy between desired and actual behaviour.

14. In a novel throwing task, negative affect was significantly higher in successful athletes, compared with failed conditions.

16. Over the course of the time trial, particularly when athletes were striving to achieve a time goal, they only made an assessment regarding their performance and rate of goal progress at the halfway mark.

The prevalence of self-reported neck pain in rugby union players in Gauteng Province, SA

16. A study among a cohort of rugby union players in Gauteng Province showed that over $50 \%$ of the players had neck pain at the time of the study.

17. Most rugby union players with neck pain were treated by a biokineticist.

18. Just over half of the rugby union players interviewed engaged in strength training specifically for their necks.

19. Neck pain caused mild to moderate disability in $18 \%$ of the players surveyed.

20. Cervical trauma occurs most frequently during the lineout. 